\title{
Association of habitual glucosamine use with risk of cardiovascular disease: prospective study in UK Biobank
}

\author{
Hao Ma, ${ }^{1,2}$ Xiang Li, ${ }^{1}$ Dianjianyi Sun, ${ }^{1}$ Tao Zhou, ${ }^{1}$ Sylvia H Ley, ${ }^{1,3,4}$ Jeanette Gustat, ${ }^{1}$ \\ Yoriko Heianza, ${ }^{1}$ Lu Qi ${ }^{1,3,4}$
}

Check for updates

${ }^{1}$ Department of Epidemiology,

School of Public Health and

Tropical Medicine, Tulane

University, New Orleans, LA

70112, USA

${ }^{2}$ The National Key Discipline, Department of Nutrition and

Food Hygiene, School of

Public Health, Harbin Medical

University, Harbin, China

${ }^{3}$ Channing Division of Network

Medicine, Department of

Medicine, Brigham and

Women's Hospital and Harvard

Medical School, Boston, MA

USA

${ }^{4}$ Department of Nutrition,

Harvard T.H. Chan School of

Public Health, Boston, MA, USA

Correspondence to: L Qi

lqi1@tulane.edu

(ORCID 0000-0002-8041-7791)

Additional material is published

online only. To view please visit

the journal online.

Cite this as: $B M J$ 2019;365:11628 http://dx.doi.org/10.1136/bmj.l1628

Accepted: 14 March 2019

\section{ABSTRACT}

\section{OBJECTIVE}

To prospectively assess the association of habitual glucosamine use with risk of cardiovascular disease (CVD) events.

\section{DESIGN}

Prospective cohort study.

SETTING

UK Biobank.

\section{PARTICIPANTS}

466039 participants without CVD at baseline who completed a questionnaire on supplement use, which included glucosamine. These participants were enrolled from 2006 to 2010 and were followed up to 2016 .

\section{MAIN OUTCOME MEASURES}

Incident CVD events, including CVD death, coronary heart disease, and stroke.

\section{RESULTS}

During a median follow-up of seven years, there were 10204 incident CVD events, 3060 CVD deaths, 5745 coronary heart disease events, and 3263 stroke events. After adjustment for age, sex, body mass index, race, lifestyle factors, dietary intakes, drug use, and other supplement use, glucosamine use was associated with a significantly lower risk of total CVD events (hazard ratio $0.85,95 \%$ confidence interval 0.80 to 0.90 ), CVD death $(0.78,0.70$ to 0.87$)$, coronary heart disease $(0.82,0.76$ to 0.88$)$, and stroke $(0.91,0.83$ to 1.00$)$. CONCLUSION

Habitual use of glucosamine supplement to relieve osteoarthritis pain might also be related to lower risks of CVD events.

\section{Introduction}

Glucosamineis anon-vitamin, non-mineral supplement widely used to relieve osteoarthritis and joint pain. ${ }^{1}$
Glucosamine is closely regulated in most European countries, where it is only sold with a prescription. However, in other countries such as the United States and Australia, it is a popular dietary supplement and approximately $20 \%$ of adults consume it daily. ${ }^{23}$

The effectiveness of glucosamine in patients with osteoarthritis and joint pain continues to be debated. ${ }^{4}$ Emerging evidence from epidemiological studies suggests that glucosamine could have a role in preventing cardiovascular disease $(\mathrm{CVD})^{3}$ and reducing mortality. ${ }^{6}$ A previous animal study reported that glucosamine extended life span by mimicking a low carbohydrate diet, ${ }^{7}$ and studies in humans have consistently shown the protective effect of a low carbohydrate diet on the development of CVD. ${ }^{8-16}$ Other animal studies have reported that the anti-inflammatory properties of glucosamine might have a preventive role in atherosclerosis development. ${ }^{17-21}$

In this prospective cohort study, we examined the association between habitual glucosamine supplement use and risk of CVD events (CVD death, coronary heart disease (CHD), and stroke) in nearly half a million adults in the UK Biobank. We also analyzed potential effect modification by other known risk factors for CVD.

\section{Methods}

Study population

The UK Biobank is a national health resource in the United Kingdom designed to improve the prevention, diagnosis, and treatment of a wide range of illnesses and to promote health throughout society. ${ }^{22} 23$ The UK Biobank recruited around 500000 participants aged 40-69 in 2006-10 from across the country.

Data from 502616 participants were available for our study. We excluded participants with CVD at baseline $(n=32187)$ and those with incomplete data on the use of glucosamine $(n=4390)$. Our final analysis included 466039 participants.

\section{WHAT IS ALREADY KNOWN ON THIS TOPIC}

Glucosamine supplement is commonly used to treat osteoarthritis, but its effectiveness in relieving osteoarthritis and joint pain continues to be debated

Recent evidence from animal studies and cross sectional studies in humans suggests that glucosamine could have a role in preventing cardiovascular disease (CVD) and reducing mortality; however, evidence from prospective studies is lacking

\section{WHAT THIS STUDY ADDS}

Habitual glucosamine use was associated with a $15 \%$ lower risk of total CVD events and a $9 \%-22 \%$ lower risk of individual CVD events (CVD death, coronary heart disease, and stroke)

These findings suggest that glucosamine might have a preventive role in the development of CVD, but further clinical trials are needed to test this hypothesis

\section{Exposure assessment}

Participants attended one of 22 assessment centers across the UK where they completed a touch screen questionnaire. One of the questions asked "Do you regularly take any of the following?", and participants could select their answer from a list of supplements, which included glucosamine. From this information, we defined glucosamine use as $0=$ no and $1=$ yes.

We used the baseline touch screen questionnaire to assess several potential confounders: age, sex, race, household income, smoking status, and alcohol intake (we calculated ethanol intake by multiplying the quantity of each type of drink -red wine, white wine, beer or cider, fortified wine, or spirits-by its standard drink size and 
reference alcohol content); self reported diabetes and high cholesterol level; drugs to treat high cholesterol, high blood pressure, and diabetes; aspirin and other nonsteroidal anti-inflammatory drug use; and dietary intakes (red meat, vegetables, fruit, fish, and cereals).

We calculated the healthy diet score by using the following factors: red meat intake up to three times each week (median); vegetable intake at least four tablespoons each day (median); fruit intake at least three pieces each day (median); fish intake at least four times each week (median); cereal intake at least five bowls each week (median); and urinary sodium concentration up to $70.6 \mathrm{mmol} / \mathrm{L}$ (median). We gave 1 point for each favorable diet factor, and the total diet score ranged from 0 to $6 .^{24}{ }^{25} \mathrm{~A}$ healthy diet was defined as a diet score of 3 or more. ${ }^{26}$

The ion selective electrode method (AU5400 analyzer, Beckman Coulter) was used to measure sodium levels in stored urine samples. The analytic range for sodium was $2-200 \mathrm{mmol} / \mathrm{L}$. Details on quality control and sample preparation have been published previously. ${ }^{27}$ Body mass index was calculated by dividing a participant's weight, measured to the nearest $0.1 \mathrm{~kg}$ using the Tanita BC-418 MA body composition analyzer (Tanita Corporation of America, IL), by the square of his or her standing height in meters, measured with a Seca 202 device (SECA, Hamburg, Germany).

According to global recommendations on physical activity for health,28 we categorized participants into two groups based on total moderate physical activity minutes each week (one vigorous physical activity minute equals two moderate physical activity minutes): $<150$ or $\geq 150 \mathrm{~min} /$ week. Hypertension was defined as a self reported history of hypertension, systolic blood pressure of $140 \mathrm{~mm} \mathrm{Hg}$ or higher, diastolic blood pressure of $90 \mathrm{~mm} \mathrm{Hg}$ or higher, or taking antihypertensive drugs. Arthritis was defined by ICD-10 (international classification of diseases, 10th revision) codes M15-M19.

\section{Genotyping and genetic risk scores}

Detailed information about genotyping and imputation in the UK Biobank has been previously published. ${ }^{29} 30$ We calculated the genetic risk scores for CHD and stroke based on previously reported genetic variants ${ }^{31}{ }^{32}: 63$ single nucleotide polymorphisms (SNPs) were used for CHD, and 27 SNPs were used for stroke (supplementary tables 1 and 2). In our analytic sample, we had data for 393771 participants to calculate CHD genetic risk score, and data for 330419 participants to calculate stroke genetic risk score by using a weighted method. ${ }^{33}$ Each SNP was recoded as 0 , 1, or 2 according to the number of risk alleles. Each SNP was multiplied by a weighted risk estimate (natural logarithm of the odds ratio) for CHD or stroke obtained from the previous genome wide association study. We then added up these products. The CHD genetic risk score ranged from 3.06 to 6.54 and the stroke genetic risk score ranged from 0.52 to 3.43. Higher scores indicate a higher genetic predisposition to CHD or stroke.

\section{Ascertainment of outcomes}

The primary outcomes for this study were CVD events: CVD death, CHD, and stroke. Secondary outcomes were individual CHD events (fatal and non-fatal) and individual stroke events (fatal and non-fatal; ischemic and hemorrhagic stroke). Information on CVD events and timing of events was collected through certified death records (until 16 February 2016) and cumulative medical records of hospital diagnoses. Additional information was collected through two repeated surveys (the first visit was completed between 12 December 2009 and 7 June 2013; the second visit between 30 April 2014 and 10 August 2017).

ICD-10 codes were used in death records, whereas ICD-10 and ICD-9 (international classification of diseases, ninth revision) codes were used in medical records. CHD was defined as ICD-9 codes 410-414 and ICD-10 codes I20-I25. Stroke was defined as ICD9 codes 430-434 and 436, and ICD-10 codes I60-I64 (ischemic stroke: ICD-9 codes 433-434, ICD-10 code I63; hemorrhagic stroke: ICD-9 codes 430-432, ICD10 codes I60-I62). CVD death was defined as ICD-10 codes I00-I99.

\section{Statistical analysis}

We compared event rates in participants who did and did not use glucosamine by using Cox proportional hazards models to calculate hazard ratios and 95\% confidence intervals. The proportional hazards assumption was tested using Schoenfeld residuals. We adjusted for several potential confounders: age, sex, and race (white European, mixed, South Asian, black, others); average total annual household income (<€18000 (\$23500; €21000), £18000£30 999, £31000-€51999, £52 000-£100 000, $>£ 100000$, and "do not know" or missing); body mass index; smoking status (current, former, never, missing); alcohol intake (g/week); physical activity (<150 or $\geq 150 \mathrm{~min} /$ week); diabetes (yes, no, or missing), hypertension (yes or no), high cholesterol (yes or no), and arthritis (yes or no); antihypertensive drugs (yes or no), lipid treatment (yes or no), insulin treatment (yes or no), aspirin use (yes or no), and non-aspirin non-steroidal anti-inflammatory drug use (yes or no); healthy diet (yes or no); vitamin supplement use (yes or no; multivitamin, folic acid, vitamin $A$, vitamin $B$, vitamin $C$, vitamin $D$, vitamin E); and mineral and other dietary supplement use (yes or no; calcium, iron, zinc, selenium, fish oil). We coded missing data as a missing indicator category for categorical variables such as smoking status, and with mean values for continuous variables.

We conducted a stratified analysis to assess potential modification effects by the following factors: sex (women or men), age $(<55$ or $\geq 55)$, body mass index (18.5-24.9, 25.0-29.9, or $\geq 30)$, physical activity ( $<150$ or $\geq 150 \mathrm{~min} /$ week), smoking (never, former, or current), healthy diet (yes or no), diabetes (yes or no), hypertension (yes or no), high cholesterol (yes or no), arthritis (yes or no), aspirin use (yes or no), and non-aspirin non-steroidal anti-inflammatory drug use 
(yes or no). We evaluated potential effect modification by modeling the cross product term of the stratifying variable with glucosamine use.

We conducted several sensitivity analyses. First, because participants who took glucosamine also tended to take other supplements more often than participants who did not take glucosamine, we did a sensitivity analysis by excluding participants who used any other supplements. Second, to minimize the influence of reverse causation, we performed a sensitivity analysis by excluding participants who developed CVD events within two years of follow-up. Third, to control the influence of genetic predisposition to CHD or stroke, we adjusted for CHD or stroke genetic risk score.

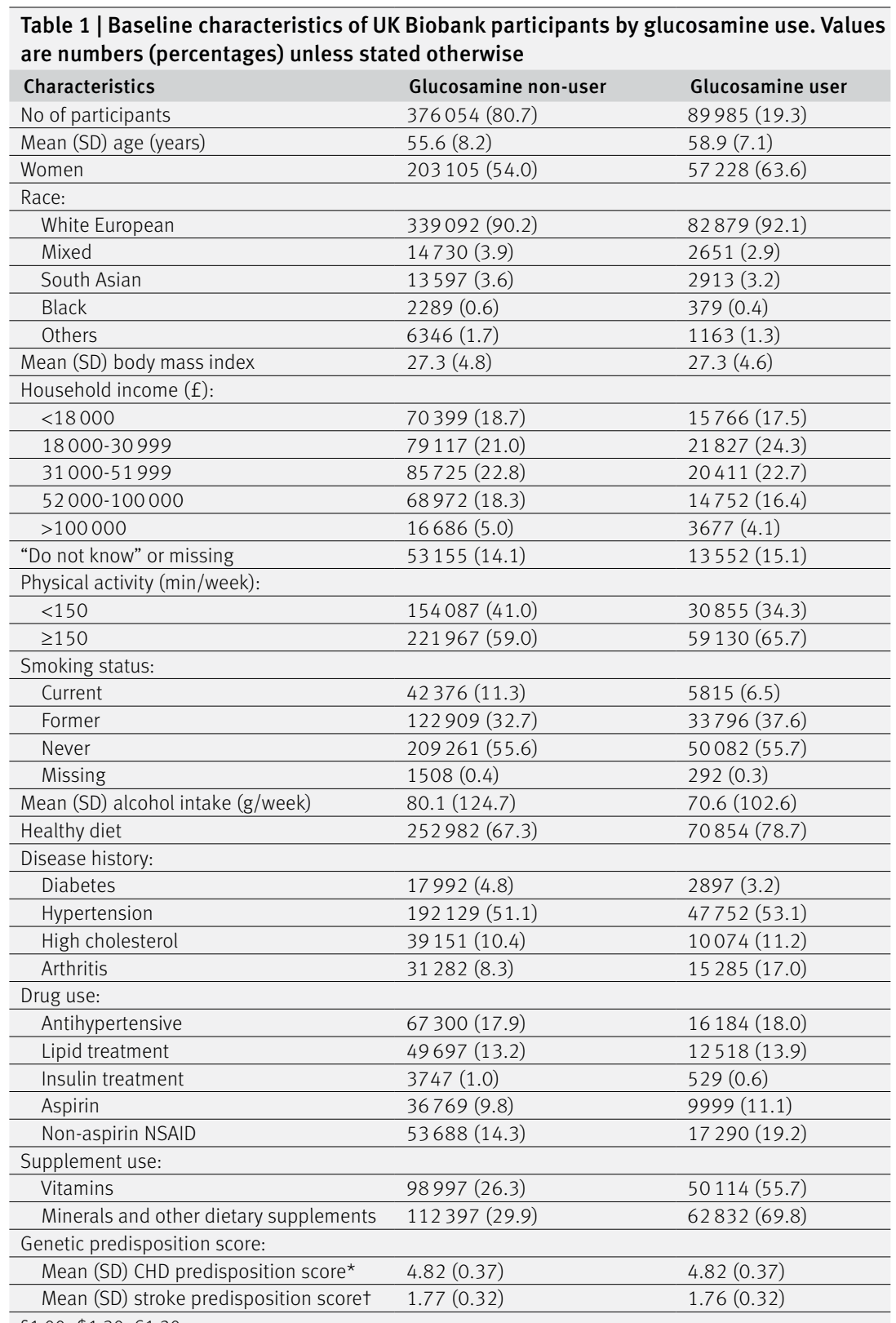

$f 1.00=\$ 1.30, € 1.20$.

$\mathrm{CHD}=$ coronary heart disease; $\mathrm{NASID}=$ non-steroidal anti-inflammatory drug.

*Data were available for 393771 participants.

tData were available for 330419 participants.
We conducted all statistical analyses by using SAS version 9.4 (SAS Institute, Cary, NC). All statistical tests were two sided, and we considered a P value less than 0.05 to be statistically significant.

\section{Patient and public involvement}

No patients were involved in setting the research question or the outcome measures, nor were they involved in the design and implementation of the study. No plans exist to disseminate the results to study participants.

\section{Results}

Table 1 shows baseline characteristics of the study participants according to the use of glucosamine. Overall, $19.3 \%$ of the study population reported glucosamine use at baseline. Compared with nonusers, glucosamine users were older, more likely to be women, not current smokers, more physically active, had a healthy diet, had a lower alcohol intake, and had a higher prevalence of hypertension, arthritis, and high cholesterol, but a lower prevalence of diabetes. Glucosamine users also tended to take more aspirin, non-aspirin non-steroidal anti-inflammatory drugs, vitamins, minerals, and other dietary supplements than non-users.

Table 2 shows the associations between glucosamine use and incident CVD events. During a median followup of seven years, we recorded 10204 incident CVD events, 3060 CVD deaths, 5745 incident CHD events, and 3263 incident stroke events. In the age adjusted analyses, we found significant inverse associations between glucosamine use and risk of total CVD events, CVD death, CHD, and stroke (all $\mathrm{P}<0.001$ ). In the multivariable adjusted analyses, the hazard ratios associated with glucosamine use were 0.85 (95\% confidence interval 0.80 to $0.90 ; \mathrm{P}<0.001)$ for total CVD events; 0.78 (0.70 to $0.87 ; \mathrm{P}<0.001)$ for CVD death; 0.82 ( 0.76 to $0.88 ; \mathrm{P}<0.001$ ) for $\mathrm{CHD}$; and 0.91 (0.83 to $1.00 ; \mathrm{P}=0.04$ ) for stroke.

We analyzed the relations between glucosamine use and subtypes of CHD and stroke. For CHD, glucosamine use was associated with significantly lower risks of non-fatal CHD (hazard ratio 0.84, 95\% confidence interval 0.77 to $0.91 ; \mathrm{P}<0.001)$ and fatal CHD (0.70, 0.59 to $0.85 ; \mathrm{P}<0.001)$. For stroke, glucosamine use was associated with a marginally significantly lower risk of non-fatal stroke $(0.91,0.82$ to $1.01 ; \mathrm{P}=0.08)$, but it was not associated with risk of fatal stroke $(0.87,0.68$ to $1.13 ; \mathrm{P}=0.30)$. There was no significant inverse association between glucosamine use and risk of ischemic stroke $(0.92,0.80$ to $1.03 ; \mathrm{P}=0.14)$ or hemorrhagic stroke $(0.89,0.75$ to $1.07 ; \mathrm{P}=0.21)$.

We conducted stratified analyses according to potential CVD risk factors. We observed consistent and significant interactions between glucosamine use and smoking on risks of CVD events and CHD (P for interaction $=0.02$ and 0.004 , respectively) (figs 1 and 2). The associations between glucosamine use and these CVD outcomes were stronger among current smokers than among former or never smokers. The associations 


\begin{tabular}{|c|c|c|c|c|c|c|}
\hline CVD event or death & Glucosamine non-user & Glucosamine user & $\begin{array}{l}\text { Age adjusted } \\
\text { hazard ratio }(95 \% \mathrm{CI})\end{array}$ & $P$ value & $\begin{array}{l}\text { Multivariate adjusted } \\
\text { hazard ratio* }(95 \% \mathrm{Cl})\end{array}$ & $P$ value \\
\hline CVD event† & $8436(2.2)$ & $1768(2.0)$ & $0.71(0.68$ to 0.75$)$ & $<0.001$ & $0.85(0.80$ to 0.90$)$ & $<0.001$ \\
\hline CVD death & $2580(0.7)$ & $480(0.5)$ & $0.61(0.55$ to 0.67$)$ & $<0.001$ & 0.78 (0.70 to 0.87$)$ & $<0.001$ \\
\hline CHD: & $4820(1.3)$ & $925(1.0)$ & $0.66(0.62$ to 0.71$)$ & $<0.001$ & $0.82(0.76$ to 0.88$)$ & $<0.001$ \\
\hline Non-fatal & $3823(1.0)$ & $776(0.9)$ & 0.71 (0.66 to 0.77$)$ & $<0.001$ & 0.84 (0.77 to 0.91) & $<0.001$ \\
\hline Fatal & $997(0.3)$ & $149(0.2)$ & $0.50(0.42$ to 0.59$)$ & $<0.001$ & $0.70(0.59$ to 0.85$)$ & $<0.001$ \\
\hline Stroke: & $2623(0.7)$ & $640(0.7)$ & $0.82(0.75$ to 0.90$)$ & $<0.001$ & $0.91(0.83$ to 1.00$)$ & 0.04 \\
\hline Non-fatal & $2271(0.6)$ & $555(0.6)$ & 0.83 (0.75 to 0.91) & $<0.001$ & $0.91(0.82$ to 1.01$)$ & 0.08 \\
\hline Fatal & $352(0.1)$ & $85(0.1)$ & 0.79 (0.62 to 1.00$)$ & 0.05 & $0.87(0.68$ to 1.13$)$ & 0.30 \\
\hline Ischemic & $1833(0.5)$ & $441(0.5)$ & 0.79 (0.71 to 0.88$)$ & $<0.001$ & $0.92(0.82$ to 1.03$)$ & 0.14 \\
\hline Hemorrhagic & $696(0.2)$ & $175(0.2)$ & $0.89(0.75$ to 1.05$)$ & 0.16 & $0.89(0.75$ to 1.07$)$ & 0.21 \\
\hline \multicolumn{7}{|c|}{$\begin{array}{l}£ 1.00=\$ 1.30, € 1.20 \text {. } \\
\text { CHD=coronary heart disease. } \\
\text { *Adjusted for age, sex, race (white European, mixed, South Asian, black, others), average total annual household income }(<£ 18000, £ 18000-£ 30999, £ 31000-£ 51999, f 52000-£ 100000 \text {, } \\
>f 100000 \text {, and “do not know” or missing), body mass index, smoking status (never, former, current, or missing), alcohol intake, physical activity ( }<150 \text { or } \geq 150 \text { min/week), diabetes (yes, no, or } \\
\text { missing), hypertension (yes or no), high cholesterol (yes or no), arthritis (yes or no), antihypertensive drugs (yes or no), lipid treatment (yes or no), insulin treatment (yes or no), aspirin use (yes or } \\
\text { no), non-aspirin non-steroidal anti-inflammatory drug use (yes or no), vitamin supplement use (yes or no), mineral and other dietary supplement use (yes or no), and healthy diet (yes or no). } \\
\text { tComposite endpoint of first major cardiovascular event (CVD death, CHD, or stroke). }\end{array}$} \\
\hline
\end{tabular}

between glucosamine use and CVD outcomes were not modified by other risk factors, including age, sex, body mass index, physical activity, healthy diet, diabetes, hypertension, high cholesterol, arthritis, and aspirin and non-aspirin non-steroidal anti-inflammatory drug use (figs 1 and 2).

In our sensitivity analyses, the associations between glucosamine use and CVD outcomes did not change appreciably: first, when we excluded participants who used any other supplements (supplementary table 3); second, when we excluded participants who developed CVD events within two years of follow-up (supplementary table 4); and third, after additional adjustment for genetic predisposition to CHD or stroke (CHD or stroke genetic risk score; supplementary table 5). In addition, we did not find significant interactions between glucosamine use and CHD genetic risk score on the risk of CHD ( $\mathrm{P}$ for interaction=0.35) or significant interaction between glucosamine use and stroke genetic risk score on the risk of stroke ( $\mathrm{P}$ for interaction $=0.35$ ).

\section{Discussion}

In this large prospective study, habitual glucosamine use was associated with a $15 \%$ lower risk of total CVD events and a 9\%-22\% lower risk of individual cardiovascular events (CVD death, CHD, and stroke). Such associations were independent of traditional risk factors, including sex, age, income, body mass index, physical activity, healthy diet, alcohol intake, smoking status, diabetes, hypertension, high cholesterol, arthritis, drug use, and other supplement use. In addition, we found that the associations between glucosamine use and CVD outcomes were statistically significantly modified by smoking status.

\section{Comparison with other studies}

Our findings are in line with several previous studies that show inverse associations of glucosamine use with CVD risk and mortality. In a cross sectional study of 266844 Australian participants, glucosamine use was found to be inversely associated with risks of heart attack or angina (odds ratio $0.79,95 \%$ confidence interval 0.73 to 0.86 ) and other heart diseases (0.82, 0.76 to 0.89$).^{3}$ In the Vitamins and Lifestyle (VITAL) cohort study, glucosamine use was significantly associated with an $18 \%$ lower risk of total mortality. ${ }^{634}$ Similarly in our study, we found that glucosamine use was consistently associated with lower risks of subtypes of CHD, including fatal and non-fatal CHD. Our lack of statistically significant associations between glucosamine use and subtypes of stroke is probably because of small numbers of participants in the subtype groups.

\section{Biological plausibility}

Several potential mechanisms could explain the observed protective relation between glucosamine use and CVD diseases. In the National Health and Nutrition Examination Survey (NHANES) study, regular use of glucosamine was associated with a statistically significant reduction in $\mathrm{C}$ reactive protein concentrations, which is a marker for systemic inflammation. ${ }^{35}$ Animal studies also reported that the anti-inflammatory properties of glucosamine might have a preventive role in the pathophysiology of CVD. ${ }^{17-21}$ In addition, a previous study found that glucosamine could mimic a low carbohydrate diet by decreasing glycolysis and increasing amino acid catabolism in mice ${ }^{7}$; therefore, glucosamine has been treated as an energy restriction mimetic agent. ${ }^{36}$ Low carbohydrate diets have been related to a reduced risk of CVD in epidemiological studies, ${ }^{89}$ and several recent diet intervention trials report that a low carbohydrate diet has a protective effect against the development of CVD. ${ }^{10-16}$ Other mechanisms might also be involved, and future investigations are needed to explore the functional roles of glucosamine in cardiovascular health.

We found consistent interactions between glucosamine use and smoking on CVD outcomes. Inverse associations of glucosamine use with CVD outcomes were stronger in current smokers than in former smokers or never smokers. We found habitual 


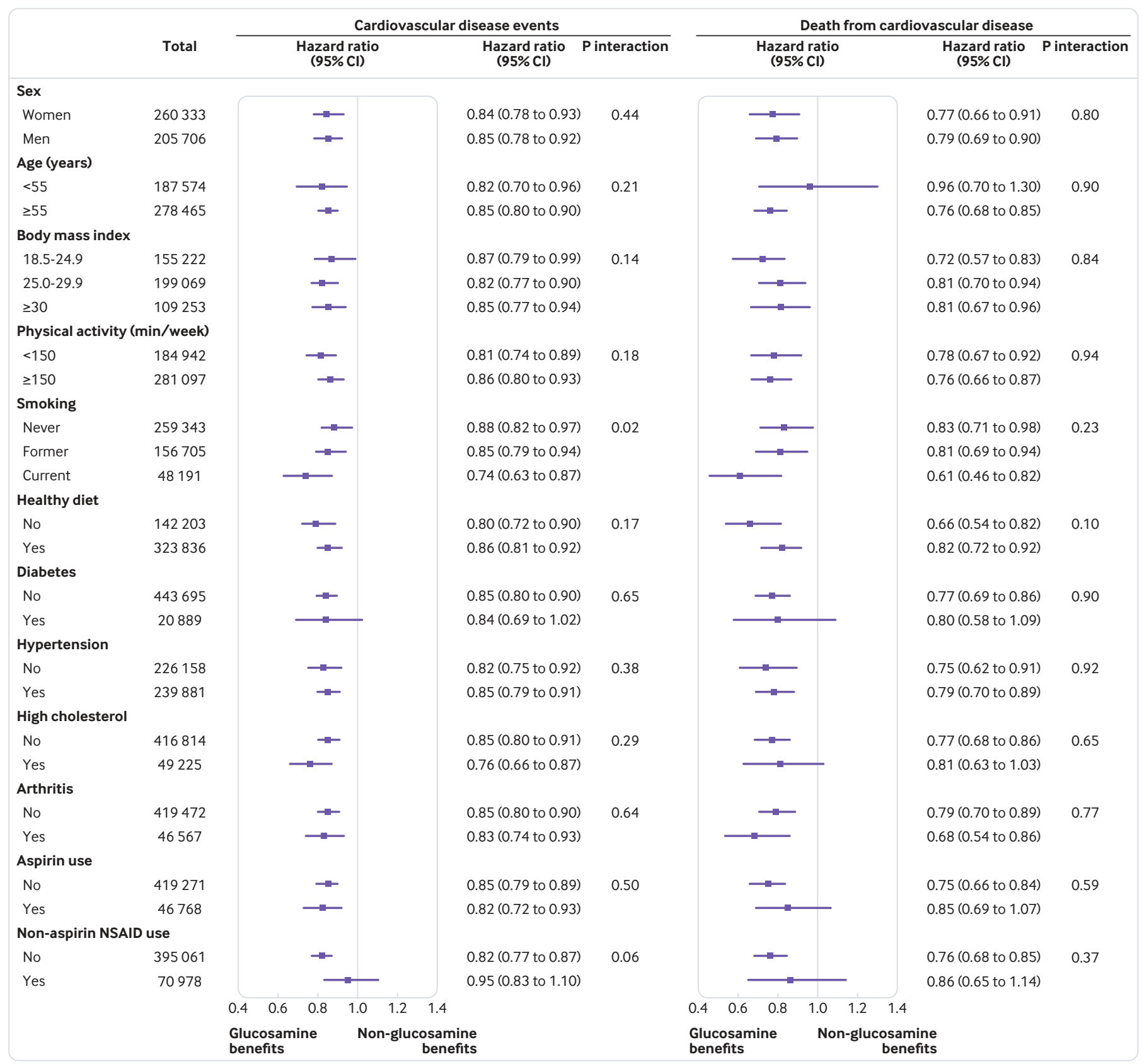

Fig 1 | Association of glucosamine supplement use and risk of cardiovascular disease event and cardiovascular disease death stratified by potential risk factors. Results were adjusted for age, sex, race (white European, mixed, South Asian, black, others), average total annual household income ( $₫ 18000, f 18000-£ 30999, f 31000-f 51999, f 52000-f 100000,>f 100000$, and "do not know" or missing; $f 1.00=\$ 1.30, € 1.20)$, alcohol intake, physical activity ( $\$ 150$ or $\geq 150 \mathrm{~min} /$ week), diabetes (yes, no, or missing), hypertension (yes or no), high cholesterol (yes or no), arthritis (yes or no), antihypertensive drugs (yes or no), lipid treatment (yes or no), insulin treatment (yes or no), aspirin use (yes or no), non-aspirin non-steroidal antiinflammatory drug (NSAID) use (yes or no), vitamin supplement use (yes or no), mineral and other dietary supplement use (yes or no), and healthy diet (yes or no)

glucosamine use was associated with a $12 \%$ and an $18 \%$ lower risk of CHD in never and former smokers, respectively, compared with a $37 \%$ lower risk in current smokers. We could not rule out the possibility that these results were due to chance. However, smokers have higher levels of inflammation and a higher risk of CVD compared with non-smokers. ${ }^{37}$ Additionally it has been hypothesized that anti-inflammatory agents might be more effective in participants with higher inflammation stress ${ }^{37}$; ; thus the interaction between glucosamine use and smoking is biologically feasible. Given the important role smoking has in the development of CVD, further studies are needed to evaluate the effect of glucosamine in CVD prevention, particularly among current smokers.

\section{Strengths and limitations of this study}

Our study has several major strengths, including the large sample size and the wealth of information on lifestyle, diet, and other covariates, which enabled 


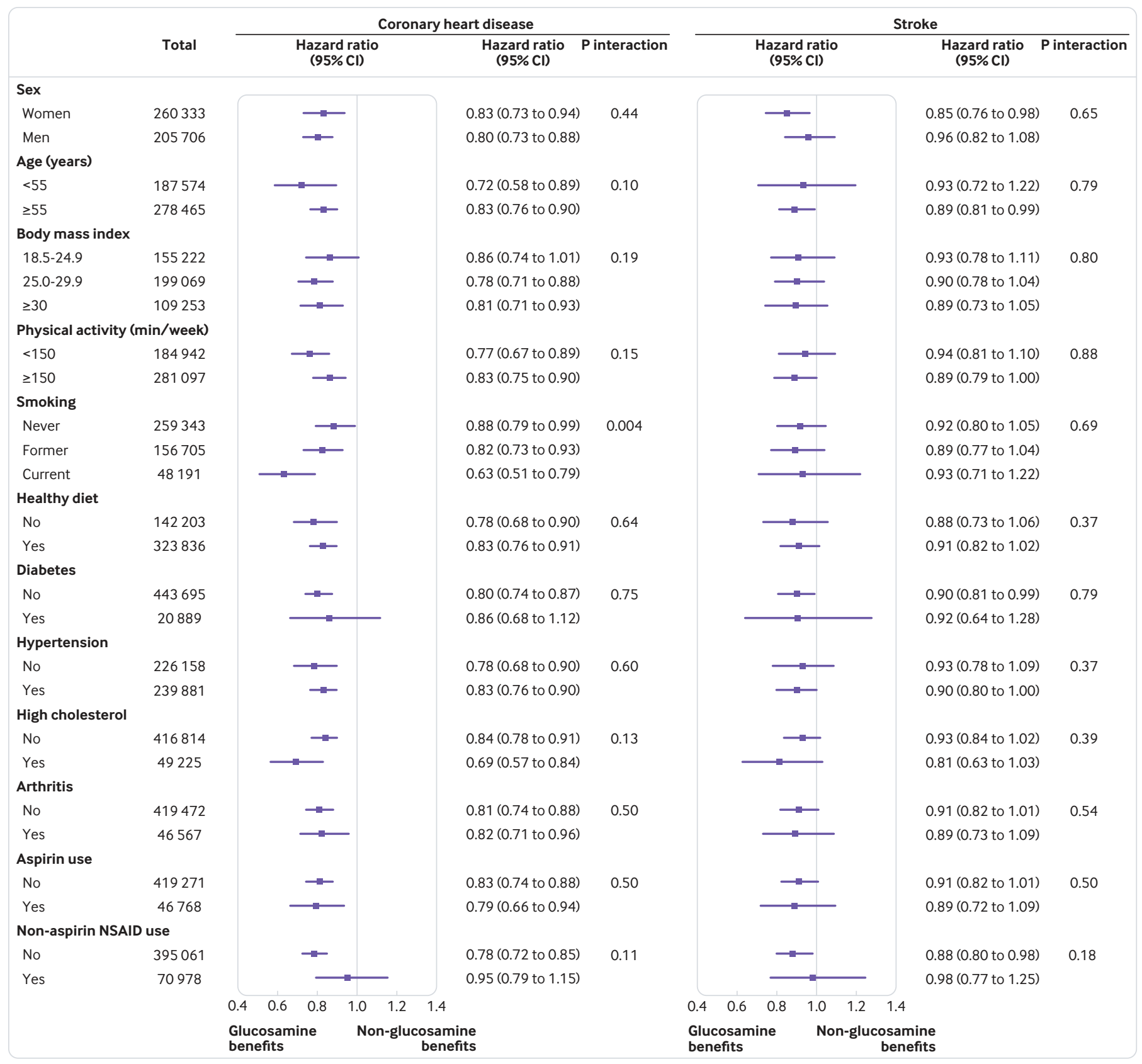

Fig 2 | Association of glucosamine supplement use and risk of coronary heart disease and stroke stratified by potential risk factors. Results were adjusted for age, sex, race (white European, mixed, South Asian, black, others), average total annual household income ( $₫ 18000, f 18000$ -

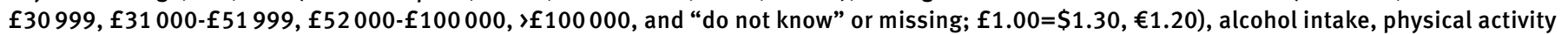
(«150 or $\geq 150 \mathrm{~min} /$ week), diabetes (yes, no, or missing), hypertension (yes or no), high cholesterol (yes or no), arthritis (yes or no), antihypertensive drugs (yes or no), lipid treatment (yes or no), insulin treatment (yes or no), aspirin use (yes or no), non-aspirin non-steroidal anti-inflammatory drug (NSAID) use (yes or no), vitamin supplement use (yes or no), mineral and other dietary supplement use (yes or no), and healthy diet (yes or no)

us to conduct comprehensive sensitivity analyses and subgroup analyses. We acknowledge that our study also has potential limitations. First, the UK Biobank did not record detailed information on glucosamine use, such as the dosage and the duration of use. Therefore, further studies are needed to investigate such associations. Second, the UK Biobank did not collect information on the side effects of glucosamine use. However, glucosamine has been rated the safest supplement for osteoarthritis, with few side effects reported, such as occasional allergic reactions, diarrhea, constipation, nausea, and heartburn. ${ }^{1}$ Although previous studies have suggested that glucosamine might worsen glucose tolerance in participants with a high risk of diabetes, ${ }^{39} 40$ clinical trials have shown that glucosamine has no effect on glucose metabolism and lipid profile at any oral dose in healthy participants and patients with diabetes..$^{41-43}$

A third limitation is that specific information on forms of glucosamine supplement (glucosamine 
sulfate, glucosamine hydrochloride, N acetyl glucosamine) was not collected, and so it was difficult to assess whether the association between various forms of glucosamine supplement and CVD risk might differ. However, most glucosamine products available on the market contain glucosamine sulfate. Fourth, it is difficult to separate the effects of a healthy lifestyle from the use of habitual supplements in an observational study. Habitual glucosamine use might be a marker for a healthy lifestyle in this study. Therefore, we could not exclude the possibility that the observed inverse associations were driven by healthy lifestyle factors among glucosamine users, although we had carefully adjusted for potential confounding in our analyses. Finally, potential reverse causality might still exist in our study, although the results remained unchanged when we excluded participants with CVD events that occurred during the first two years of follow-up.

\section{Conclusions}

Habitual use of glucosamine supplement to relieve osteoarthritis pain might also be related to lower risks of CVD events. Further clinical trials are needed to test this hypothesis.

Contributors: LQ and HM conceived and designed the study. HM performed the statistical analysis. HM and LQ wrote the first draft of the manuscript. All the authors participated in the interpretation of the results and critical revision of the manuscript. LQ had full access to all of the data in the study and takes responsibility for the integrity of the data and the accuracy of the data analysis; he is the guarantor. The corresponding author attests that all listed authors meet authorship criteria and that no others meeting the criteria have been omitted.

Funding: LQ was supported by grants from the National Heart, Lung, and Blood Institute (HL071981, HL034594, HL126024) and the National Institute of Diabetes and Digestive and Kidney Diseases (DK115679, DK091718, DK100383, DK078616). SHL is partially supported by grant P20GM109036 from the National Institute of General Medical Sciences of the National Institutes of Health.

Competing interests: All authors have completed the ICMJE uniform disclosure form at www.icmje.org/coi_disclosure.pdf and declare: no support from any organization for the submitted work; no financial relationships with any organizations that might have an interest in the submitted work in the previous three years; no other relationships or activities that could appear to have influenced the submitted work.

Ethical approval: The UK Biobank study was approved by the National Health Service's National Research Ethics Service. The present analysis was approved by the institutional review board of Tulane University (New Orleans, LA).

Data sharing: The genetic and phenotypic UK Biobank data are available on application to the UK Biobank (www.ukbiobank.ac.uk/).

Transparency: The lead author (LQ) affirms that the manuscript is an honest, accurate, and transparent account of the study being reported; that no important aspects of the study have been omitted; and that any discrepancies from the study as planned have been explained.

This is an Open Access article distributed in accordance with the Creative Commons Attribution Non Commercial (CC BY-NC 4.0) license, which permits others to distribute, remix, adapt, build upon this work non-commercially, and license their derivative works on different terms, provided the original work is properly cited and the use is non-commercial. See: http://creativecommons.org/licenses/ by-nc/4.0/

1 Jordan KM, Arden NK, Doherty M, et al, Standing Committee for International Clinical Studies Including Therapeutic Trials ESCISIT. EULAR Recommendations 2003: an evidence based approach to the management of knee osteoarthritis: Report of a Task Force of the Standing Committee for International Clinical Studies Including Therapeutic Trials (ESCISIT). Ann Rheum Dis 2003;62:1145-55. doi:10.1136/ard.2003.011742
2 Barnes PM, Bloom B, Nahin RL. Complementary and alternative medicine use among adults and children: United States, 2007. Natl Health Stat Report 2008;12:1-23.

3 Sibbritt D, Adams J, Lui CW, Broom A, Wardle J. Who uses glucosamine and why? A study of 266,848 Australians aged 45 years and older. PLoS One 2012;7:e41540. doi:10.1371/journal. pone.0041540

4 Clegg DO, Reda DJ, Harris CL, et al. Glucosamine, chondroitin sulfate, and the two in combination for painful knee osteoarthritis. N Engl s Med 2006;354:795-808. doi:10.1056/NEJMoa052771

5 Wilkens P, Scheel IB, Grundnes O, Hellum C, Storheim K. Effect of glucosamine on pain-related disability in patients with chronic low back pain and degenerative lumbar osteoarthritis: a randomized controlled trial. JAMA 2010;304:45-52. doi:10.1001/ jama.2010.893

6 Bell GA, Kantor ED, Lampe JW, Shen DD, White E. Use of glucosamine and chondroitin in relation to mortality. Eur J Epidemiol 2012;27:593-603. doi:10.1007/s10654-012-9714-6

7 Weimer S, Priebs J, Kuhlow D, et al. D-Glucosamine supplementation extends life span of nematodes and of ageing mice. Nat Commun 2014;5:3563. doi:10.1038/ncomms4563

8 Hu T, Bazzano LA. The low-carbohydrate diet and cardiovascular risk factors: evidence from epidemiologic studies. Nutr Metab Cardiovas Dis 2014;24:337-43. doi:10.1016/j.numecd.2013.12.008

9 Halton TL, Willett WC, Liu S, et al. Low-carbohydrate-diet score and the risk of coronary heart disease in women. $N$ Englf Med 2006;355:1991-2002. doi:10.1056/NEJMoa055317

10 Bazzano LA, Hu T, Reynolds K, et al. Effects of low-carbohydrate and low-fat diets: a randomized trial. Ann Intern Med 2014;161:309-18. doi:10.7326/M14-0180

11 Foster GD, Wyatt HR, Hill JO, et al. A randomized trial of a lowcarbohydrate diet for obesity. N Engl J Med 2003;348:2082-90. doi:10.1056/NEJMoa022207

12 Shai I, Schwarzfuchs D, Henkin Y, et al, Dietary Intervention Randomized Controlled Trial (DIRECT) Group. Weight loss with a low-carbohydrate, Mediterranean, or low-fat diet. N Engl] Med 2008;359:229-41. doi:10.1056/NEJMoa0708681

13 Dansinger ML, Gleason JA, Griffith JL, Selker HP, Schaefer EJ. Comparison of the Atkins, Ornish, Weight Watchers, and Zone diets for weight loss and heart disease risk reduction: a randomized trial. JAMA 2005;293:43-53. doi:10.1001/jama.293.1.43

14 Foster GD, Wyatt HR, Hill JO, et al. Weight and metabolic outcomes after 2 years on a low-carbohydrate versus low-fat diet: a randomized trial. Ann Intern Med 2010;153:147-57. doi:10.7326/0003-4819153-3-201008030-00005

15 Yancy WSIr, Olsen MK, Guyton JR, Bakst RP, Westman EC. A lowcarbohydrate, ketogenic diet versus a low-fat diet to treat obesity and hyperlipidemia: a randomized, controlled trial. Ann Intern Med 2004:140:769-77. doi:10.7326/0003-4819-140-10200405180-00006

16 Stern L, Iqbal N, Seshadri P, et al. The effects of low-carbohydrate versus conventional weight loss diets in severely obese adults: oneyear follow-up of a randomized trial. Ann Intern Med 2004:140:778 85. doi:10.7326/0003-4819-140-10-200405180-00007

17 Zou L, Yang S, Champattanachai V, et al. Glucosamine improves cardiac function following trauma-hemorrhage by increased protein O-GlcNAcylation and attenuation of NF-kB signaling. Am J Physiol Heart Circ Physiol 2009;296:H515-23. doi:10.1152/ ajpheart.01025.2008

18 Liu J, Marchase RB, Chatham JC. Increased O-GlcNAc levels during reperfusion lead to improved functional recovery and reduced calpain proteolysis. Am J Physiol Heart Circ Physiol 2007;293:H1391-9. doi:10.1152/ajpheart.00285.2007

19 Xing D, Feng W, Nöt LG, et al. Increased protein O-GIcNAc modification inhibits inflammatory and neointimal responses to acute endoluminal arterial injury. Am I Physiol Heart Circ Physiol 2008;295:H335-42. doi:10.1152/ajpheart.01259.2007

20 Duan W, Paka L, Pillarisetti S. Distinct effects of glucose and glucosamine on vascular endothelial and smooth muscle cells: evidence for a protective role for glucosamine in atherosclerosis. Cardiovasc Diabetol 2005;4:16. doi:10.1186/1475-2840-4-16

21 Yao D, Xu L, Xu O, et al. O-Linked B-N-Acetylglucosamine Modification of A20 Enhances the Inhibition of NF-kB (Nuclear Factor-kB) Activation and Elicits Vascular Protection After Acute Endoluminal Arterial Injury. Arterioscler Thromb Vasc Biol 2018;38:1309-20.

22 Sudlow C, Gallacher J, Allen N, et al. UK biobank: an open access resource for identifying the causes of a wide range of complex diseases of middle and old age. PLoS Med 2015;12:e1001779. doi:10.1371/journal.pmed.1001779

23 Fry A, Littlejohns TJ, Sudlow C, et al. Comparison of sociodemographic and health-related characteristics of UK Biobank participants with those of the general population. Am J Epidemiol 2017;186:1026-34. doi:10.1093/aje/kwx246

24 Lloyd-Jones DM, Hong Y, Labarthe D, et al, American Heart Association Strategic Planning Task Force and Statistics 
Committee. Defining and setting national goals for cardiovascular health promotion and disease reduction: the American Heart Association's strategic Impact Goal through 2020 and beyond. Circulation 2010;121:586-613. doi:10.1161/ CIRCULATIONAHA.109.192703

25 Pazoki R, Dehghan A, Evangelou E, et al. Genetic predisposition to high blood pressure and lifestyle factors: associations with midlife blood pressure levels and cardiovascular events. Circulation 2018;137:653-61. doi:10.1161/ CIRCULATIONAHA.117.030898

26 Mozaffarian D. Dietary and policy priorities for cardiovascular disease, diabetes, and obesity: a comprehensive review. Circulation 2016:133:187-225.

27 Elliott P, Peakman TCUK Biobank. The UK Biobank sample handling and storage protocol for the collection, processing and archiving of human blood and urine. Int J Epidemiol 2008;37:234-44. doi:10.1093/ije/dym276

28 World Health Organization. Global recommendations on physical activity for health. 2010, 2015, WHO.

29 Biobank UK. Genotype imputation and genetic association studies of UK Biobank. Interim Data Release, 2015: 11.

30 Bycroft C, Freeman C, Petkova D, et al. Genome-wide genetic data on 500000 UK Biobank participants. bioRxiv 2017; published online 20 Jul.

31 Nikpay M, Goel A, Won HH, et al. A comprehensive 1,000 Genomesbased genome-wide association meta-analysis of coronary artery disease. Nat Genet 2015;47:1121-30. doi:10.1038/ng.3396

32 Malik R, Chauhan G, Traylor M, et al, AFGen Consortium, Cohorts for Heart and Aging Research in Genomic Epidemiology (CHARGE) Consortium, International Genomics of Blood Pressure (iGENBP) Consortium, INVENT Consortium, STARNET, BioBank Japan Cooperative Hospital Group, COMPASS Consortium, EPIC-CVD Consortium, EPIC-InterAct Consortium, International Stroke Genetics Consortium (ISGC), METASTROKE Consortium, Neurology Working Group of the CHARGE Consortium, NINDS Stroke Genetics Network (SiGN), UK Young Lacunar DNA Study, MEGASTROKE Consortium, MEGASTROKE Consortium. Multiancestry genome-wide association study of 520,000 subjects identifies 32 loci associated with stroke and stroke subtypes. Nat Genet 2018;50:524-37. doi:10.1038/ s41588-018-0058-3

33 Ripatti S, Tikkanen E, Orho-Melander M, et al. A multilocus genetic risk score for coronary heart disease: case-control and prospective cohort analyses. Lancet 2010;376:1393-400. doi:10.1016/S01406736(10)61267-6

34 Pocobelli G, Kristal AR, Patterson RE, et al. Total mortality risk in relation to use of less-common dietary supplements. Am J Clin Nutr 2010;91:1791-800. doi:10.3945/ajcn.2009.28639

35 Kantor ED, Lampe JW, Vaughan TL, Peters U, Rehm CD, White E. Association between use of specialty dietary supplements and C-reactive protein concentrations. Am J Epidemiol 2012;176:1002 13. doi:10.1093/aje/kws186

36 Nikolai S, Pallauf K, Huebbe P, Rimbach G. Energy restriction and potential energy restriction mimetics. Nutr Res Rev 2015;28:100-20. doi:10.1017/S0954422415000062

37 Ridker PM, Cushman M, Stampfer MJ, Tracy RP, Hennekens CH. Inflammation, aspirin, and the risk of cardiovascular diseas in apparently healthy men. N Engl J Med 1997;336:973-9. doi:10.1056/NEIM199704033361401

38 Bardia A, Ebbert JO, Vierkant RA, et al. Association of aspirin and nonaspirin nonsteroidal anti-inflammatory drugs with cancer incidence and mortality. J Natl Cancer Inst 2007;99:881-9. doi:10.1093/jnci/djk200

39 Pham T, Cornea A, Blick KE, Jenkins A, Scofield RH. Oral glucosamine in doses used to treat osteoarthritis worsens insulin resistance. Am Med Sci 2007:333:333-9. doi:10.1097/MAl.0b013e318065bdbe

40 Biggee BA, Blinn CM, Nuite M, Silbert JE, McAlindon TE. Effects of oral glucosamine sulphate on serum glucose and insulin during an oral glucose tolerance test of subjects with osteoarthritis. Ann Rheum Dis 2007;66:260-2. doi:10.1136/ard.2006.058222

41 Scroggie DA, Albright A, Harris MD. The effect of glucosaminechondroitin supplementation on glycosylated hemoglobin levels in patients with type 2 diabetes mellitus: a placebo-controlled, doubleblinded, randomized clinical trial. Arch Intern Med 2003;163:158790. doi:10.1001/archinte.163.13.1587

42 Albert SG, Oiknine RF, Parseghian S, Mooradian AD, Haas MJ, McPherson T. The effect of glucosamine on Serum HDL cholesterol and apolipoprotein Al levels in people with diabetes. Diabetes Care 2007:30:2800-3. doi:10.2337/dc07-0545

43 Simon RR, Marks V, Leeds AR, Anderson JW. A comprehensive review of oral glucosamine use and effects on glucose metabolism in normal and diabetic individuals. Diabetes Metab Res Rev 2011;27:14-27. doi:10.1002/dmrr.1150

Supplementary information: additional tables 1-5 